\title{
A SEARCH FOR CORONAL EMISSION LINES FROM EARLY-TYPE STARS*
}

\author{
D. Baade and L.B. Lucy \\ European Southern Observatory \\ Karl-Schwarzschild-Str. 2 \\ D-8046 Garching, Fed. Rep. Germany
}

\section{INTRODUCTION}

For more than ten years now, a controversial issue in studies of stellar winds has concerned the existence or not of a coronal zone $\left(T \sim 10^{6} \mathrm{~K}\right)$ at the base of the cool winds $\left(T \sim T_{\text {eff }}\right)$ of early-type stars. The latest revival of interest in this possibility is due to Wolfire et al. (1985) who showed that Waldron's (1984) recombination stellar wind (RSW) version of the hot corona - cool wind model (Hearn 1975; Cassinelli et al. 1978) yields models for $\varsigma$ Puppis (O4 If) that are consistent with both IRAS and Einstein IPC data, thus refuting an earlier claim (Lamers et al. 1984) to have excluded the existence of a coronal zone.

\section{ZETA PUPPIS}

Stimulated by this work of Wolfire et al. (1985), we have repeated with greater sensitivity the search for coronal line emission previously attempted at the Washburn Observatory (Nordsieck et al. 1981). In our initial investigation, we concentrated on $\varsigma$ Puppis because the narrow range of permissable coronal parameters (Wolfire et al. 1985) allowed a decisive experiment to be carried out for this prototypical and much-studied example of an earlytype star with a high velocity, massive wind.

With the coronal parameters that Wolfire et al. (1985) obtain by fitting the Einstein IPC data for $\varsigma$ Puppis, the strongest coronal line should be that of [Fe XIV] at $\lambda 5303$ $\AA$, the expected equivalent width being $\sim 5 \mathrm{~m} \AA$. In the event, no signal was detected, even though the excellent, high S/N data obtaıned with the ESO CES+Reticon system would have allowed a statistically significant detection down to $\sim 1 \mathrm{~m} \AA$, the exact limit depending on the assumed kinematic properties of the coronal zone - see Baade and Lucy (1987). Accordingly, our investigation of Puppis excludes a coronal zone with the parameters determined by Wolfire et al. (1985). Moreover, given that our detection limit is substantially lower than the predicted signal, it now seems highly unlikely that a coronal structure can be found that accounts for the observed X-ray emission and is at the same time consistent with our limits on $[\mathrm{Fe}$ XIV] $\lambda 5303 \AA$ emission.

Because full details of our investigation of $\varsigma$ Puppis have now been published (Baade and Lucy 1987), further discussion here is not necessary except perhaps to emphasize that the coronal line strength calculations used to interpret our data were not carried out in the low density limit. Thus, in addition to radiative de-excitations, we included also those due

*Based on observations obtained at the European Southern Observatory, La Silla, Chile 
to electron and proton collision (Mason 1975). We also allowed for the weakening of the emission line due to the formation of an underlying absorption line.

\section{OTHER STARS}

Even though coronae are often said not to be expected for early-type stars because of the absence of a surface convection zone, violent motions observed in their reversing layers (e.g. Slettebak 1956) imply that a flux of mechanical energy is certainly available for dissipative heating. Moreover, a long-standing puzzle concerning the Copernicus UV spectrum of $\tau$ Sco can perhaps be resolved by postulating a base coronal zone. The observation is of inward moving cool gas (Lamers and Rogerson 1978), a surprising result since cool gas can be, and is in fact seen to be accelerated away from the star by radiative forces. However, if there is a coronal zone, the transition to a cool, radiatively-driven flow probably occurs at subsonic velocities (Baade and Lucy 1987) in which case the transition will be subject to the Rayleigh-Taylor instability. One can then imagine that this results in the formation of blobs of cool gas that are able to fall through the coronal gas because they are optically thick in the UV resonance lines. The constraint that the observed red-shifted absorption is non-saturated is met by the small expected covering factor of the stellar disk by the blobs.

Because the existence of base coronal zones in some early-type stars is thus not implausible, we have been motivated to continue our search for coronal line emission. Thus far data has been acquired but not yet analyzed for the stars $\tau$ Sco (B0 V), $\theta$ Car (B0 Vp), $\epsilon$ Ori (B0 Iae), $\kappa$ Ori (B0.5 Iae), $\beta$ Cru (B0.5 III), $\beta$ Cen (B1 III), $\alpha$ Lup (B1.5 III), $\epsilon$ CMa (B2 III), and $\beta$ Ori (B8 Iae).

Preliminary inspection indicates that the low detection limits achieved for $\varsigma$ Puppis probably will not be reached: in the cooler stars the problem with line blending is quite severe, and the spectra require a more careful treatment of instrumental effects.

\section{REFERENCES}

Baade, D., Lucy, L.B.: 1987, Astron. Astrophys. 178, 213.

Hearn, A.G.: 1975, Astron. Astrophys. 40, 277.

Lamers, H.J.G.L.M., Rogerson, J.B.: 1978, Astron. Astrophys. 66, 417.

Lamers, H.J.G.L.M., Waters, L.B.F.M., Wesselius, P.R.: 1984, A stron. Astrophys. 134, L17.

Mason, H.E.: 1975, Mon. Not. R. ast. Soc. 170, 651.

Nordsieck, K.H., Cassinelli, J.P., Anderson, C.M.: 1981, Astrophys. J. 248, 678.

Slettebak, A.: 1956, Astrophys. J. 124, 173.

Waldron, W.L.: 1984, Astrophys. J. 282, 256.

Wolfire, M.G., Waldron, W.L., Cassinelli, J.P.: 1985, Astron. Astrophys. 142, L25. 


\section{DISCUSSION}

DOAZAN You tried to detect the [Fe XIV] 5303 \& line for diagnosing the existence of a hot corona $(\simeq 10 \% \mathrm{~K})$ at the base of the wind of (Pup. But what about the density at which such a line may be formed?

In the solar corona, this forbidden line is formed at a density of $\simeq 100$ $\mathrm{Cm}^{-3}$ and $\mathrm{Te} \simeq 100 \mathrm{~K}$. In $\zeta$ Pup, the wind is much more massive ( $\mathrm{dy} / \mathrm{dt} \simeq 10-0$ *o yr-1), i.e. about 100 times higher than in the sun. The generally quoted value for $\mathrm{Ne}$ in the wind of $\zeta$ Pup is $\simeq 1011 \mathrm{~cm}^{-3}$ (see for ex. Hamann's (Pup model). Therefore, at the base of the wind, just after the photosphere, the density would be at least $\simeq 1012 \mathrm{~cm}-3$. Do you expect this [Fe XIV] forbidden line to be formed at such densities?

BAADE Mason (1975) has tabulated the emission rates for [Fe XIV] $\lambda 5303$ in dependence on both electron density, Ne, and electron temperature, Te. It can be seen from his work that increasing Ne from 107 $\mathrm{Cm}^{-3}$ to $1012 \mathrm{~cm}^{-3}$ at constant coronal Te actually increases the emission rate by a factor of $100-107$. Note that our predicted equivalent widths for the [FeXrv] $\lambda 5303$ line are not obtained from the emisgivity in the solar corona but by solving the equations of statistical equilibrium and radiative transfer for the various coronal models considered for $\zeta$ Pup. Thus, following Mason (1975), we adopt a 12-level model atom for FexIV and include the effects of proton collisions within the ground configuration.

APPENZELLIER How well is the critical density of [Fe XIV] $\lambda$ 5303 really known and on what work is the number which was just quoted based on ?

DOAZAN I do not quote any particular work. I only quote what is known for the Sun, where the [Fe XIV] coronal line is formed at a density of $\simeq 108 \mathrm{~cm}^{-3}$ and $\mathrm{Te} \simeq 100 \mathrm{~K}$ ( see for ex. Mihalas book on Stellar Atmospheres ). 